\title{
Search for the event horizon by means of optical observations with high temporal resolution
}

\author{
G. Beskin, ${ }^{1}$ V. Debur,${ }^{1}$ S. Karpov,${ }^{1}$ V. Plokhotnichenko ${ }^{1}$ \\ and A. Biryukov ${ }^{2}$ \\ ${ }^{1}$ Special Astrophysical Observatory of Russian Academy of Sciences, Nizhniy Arkhyz, \\ Karachaevo-Cherkessia, Russia \\ ${ }^{2}$ Sternberg Astronomical Institute of Moscow State University, Moscow, Russia
}

\begin{abstract}
The critical property of the black hole is the presence of the event horizon. It may be detected only by means of the detailed study of the emission features of its surroundings. The temporal resolution of such observations has to be better than $\sim \mathrm{rg} / \mathrm{c}$, and it lies in the $10^{-6}-10 \mathrm{~s}$ range depending on the black hole mass. In SAO RAS we have developed the MANIA hardware and software complex based on the panoramic photon counter and use it in observations on $6 \mathrm{~m}$ telescope for the search and investigation of the optical variability on the time scales of $10^{-6}-10^{3} \mathrm{~s}$ of various astronomical objects. We present the hardware and methods used for these photometrical, spectroscopic and polarimetrical observations, the principles and criteria of the object selection. The list of the latter includes objects with featureless optical spectra (DC white dwarfs, blazars) and long microlensing events.

We present the results of the observations of two objects-candidates - long MACHO event MACHO-1999-BLG-22 and radio-loud x-ray source with featureless optical spectrum J1942+10 - on the 6-m telescope in June-July 2006.
\end{abstract}

Keywords. Accretion - black hole physics - methods: data analysis - instrumentation: photometers - instrumentation: polarimeters - instrumentation: spectrographs

\section{Introduction}

Even though more than 60 years have passed since the theoretical prediction of black holes as an astrophysical objects (Oppenheimer \& Snyder 1939) in some sense they have not been discovered yet. To identify an object as a black hole, one needs to show that its mass exceeds $3 M_{\odot}$, its size is close to $r_{g}=2 G M / c^{2}$ and it has an event horizon instead of a normal surface - the distinguishing property of black holes which separates them from massive compact objects of finite size in some theories of gravity (Will 1998). However, only the two former criteria are used now for selection of black hole candidates of two types: a) with masses of 5-18 $M_{\odot}$, in X-ray binaries (see, for example, Greiner et al. 2001 ); and b) supermassive black holes in galaxy nuclei with masses of $10^{6}-10^{10} M_{\odot}$ (Shields 1999). Existence of the event horizon in such objects is usually implied by the absence of periodic pulsations of the X-ray emission from strong regular magnetic fields (the black hole "no-hair" theorem) and I type X-ray flares due to thermonuclear bursts of the accreted matter on the surface of the neutron star. High accretion rates in Xray binaries and active galactic nuclei result in the screening of regions close to the event horizon, and the most luminous parts of accretion flow are situated at distances of $10-100 r_{g}$ (Chakrabarti 1996, Cherepaschuk 2003) where general relativity effects are negligible. 

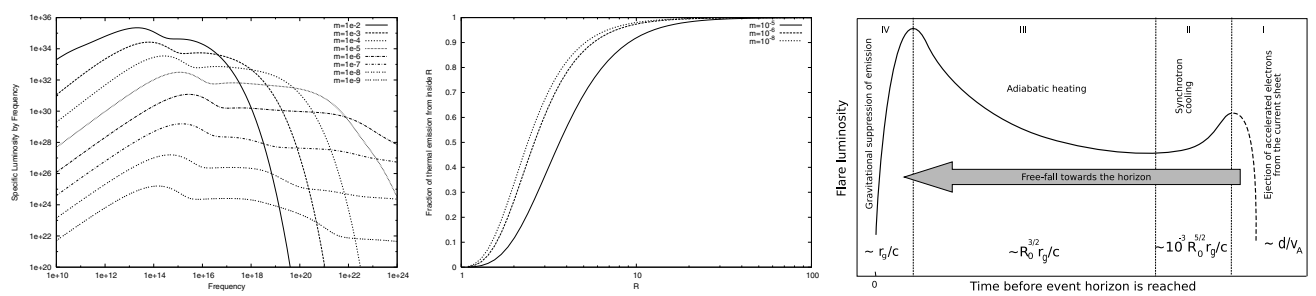

Figure 1. Properties of the isolated black hole emission. Left panel - spectra for various accretion rates, middle panel - the ratio of emission generated inside given radius, right panel - the temporal structure of the emission flare.

\section{Search for event horizon in optics}

At the same time, single stellar-mass black holes, which accrete interstellar medium of low density $\left(10^{-2}-1\right.$ per $\left.\mathrm{cm}^{3}\right)$, are the ideal case for detection and study of the event horizon. Shvartsman 1971 first demonstrated that an emitting halo of accreted matter forms around such objects and generates optical featureless emission. For the majority of Galaxy, filled with hot and warm ionized hydrogen, the regime of accretion onto the stellar mass isolated black holes is spherical, as the captured specific angular momentum is much smaller than one on the last stable orbit. The accretion rate is also small, $\dot{m}=$ $\dot{M} / \dot{M}_{e d d} \sim 10^{-10}-10^{-5}$ for the velocity of 50-100 km/s (Shvartsman 1971, Ipser \& Price 1982). So, the emission of the accretion flow is dominated by the non-thermal synchrotron component due to accelerated particles(Beskin \& Karpov 2005) (see Fig. 1). The total luminosity of such object is then $L=9.6 \cdot 10^{33} M_{10}^{3} n_{1}^{2}\left(V^{2}+c_{s}^{2}\right)_{16}^{-3} \sim 10^{29}-10^{34} \mathrm{erg} / \mathrm{s}$. The majority of such emission comes from the regions near the horizon at $(2-5) r_{g}$ (see Fig. 1).

The most striking property of the accretion flow onto the single black hole is its inhomogeneity - the clots of plasma act as a probe testing the space-time properties near the horizon. The characteristic timescale of emission variability is $\tau_{v} \sim r_{g} / c \sim 10^{-4}-10^{-5}$ sec and such short stochastic variability may be considered as a distinctive property of black hole as the smallest possible physical object with a given mass. Its parameters spectra, energy distribution and light curves - carry important information on space-time properties of the horizon (Beskin \& Karpov 2005) (see Fig. 1).

The general observational appearance of a single stellar-mass black hole at typical interstellar medium densities is the same as other optical objects without spectral lines DC-dwarfs and ROCOSes (Radio Objects with Continuous Optical Spectra, a subclass of blazars) (Pustilnik 1977). The suggestion that isolated BHs can be among them is the basis of the observational programme of search for isolated stellar-mass black holes MANIA (Multichannel Analysis of Nanosecond Intensity Alterations). It uses photometric observations of candidate objects with high time resolution, special hardware and data analysis methods (Shvartsman 1977, Beskin et al. 1997), and is based on the fact that the fast variability is the critical property of isolated black hole emission.

In observations using the 6-meter telescope of the Special Astrophysical Observatory and the standard high time resolution photometer based on photomultipliers of 40 DC-dwarfs and ROCOSes, only upper limits for variability levels of $20 \%-5 \%$ on the timescales of $10^{-6}-10 \mathrm{sec}$, respectively, were obtained, i.e. BHs were not detected (Shvartsman et al. 1989a, Shvartsman et al. 1989b, Beskin et al. 1997).

Recently, some evidences appeared that single stellar-mass black holes may be found among the stationary unidentified gamma-ray sources (Gehrels et al. 2002), gravitational lenses causing long-lasting MACHO events (Bennett et al. 2001), white dwarf - black 

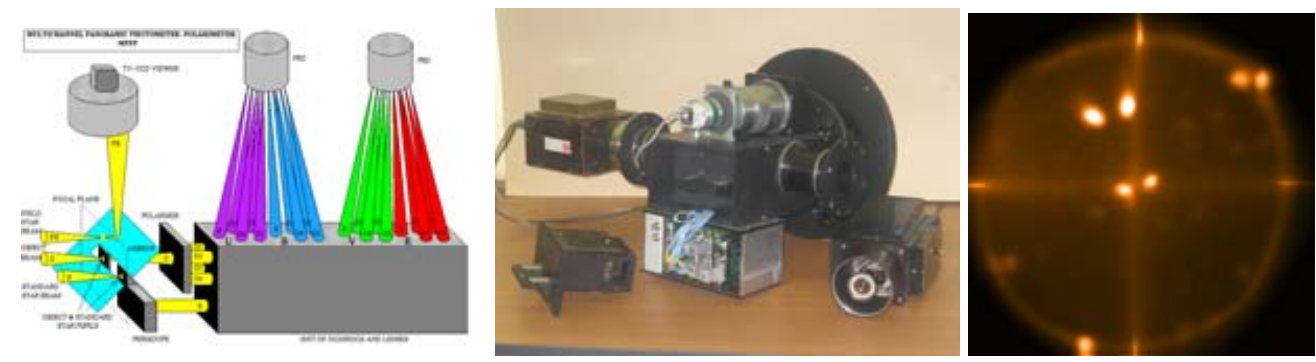

Figure 2. The scheme and the image of multichannel panoramic spectropolarimeter, and the sample star field observed in one-color photopolarimetric mode.
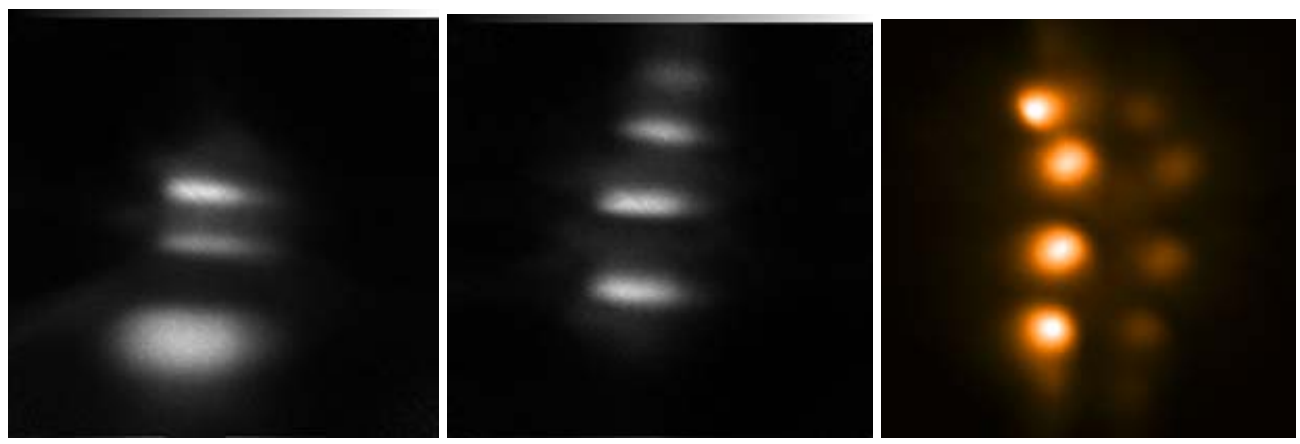

Figure 3. The different modes of MPSP operation - spectroscopic (left panel), spectropolarimetric (middle panel) and multicolor photopolarimetric (right panel).

hole binaries detected by means of self-microlensing flashes (Beskin \& Tuntsov 2002), and radio-loud x-ray sources with featureless optical spectra (Tsarevsky 2005).

To study such a faint objects (down to $22^{\mathrm{m}}$ ) we have developed the multichannel panoramic spectro-polarimeter (MPSP) based on position-sensitive detector (PSD) with $1 \mu \mathrm{s}$ time resolution (Debur et al. 2003, Plokhotnichenko et al. 2003) (see Fig. 2). Such detectors use the set of multichannel plates (MCP) for electron multiplication, and multielectrode collector to determine its position. PSD used in our observations has the following parameters: quantum efficiency of $10 \%$ in the 3700-7500 A range, (S20 photocathode), MCP stack gain of $10^{6}$, spatial resolution of $70 \mu \mathrm{m}\left(0.21^{\prime \prime}\right.$ for the 6 -m telescope), $700 \mathrm{~ns}$ time resolution, $7 \cdot 10^{4}$ pixels with $22 \mathrm{~mm}$ working diameter, and the $200-500$ counts/s detector noise. The acquisition system used is the "Quantochron 4-480" spectral time-code convertor with $30 \mathrm{~ns}$ time resolution and $10^{6}$ counts/s maximal count rate.

MPSP has the following modes of operation (see Fig. 3): one-color (U, B, V, R) photometry and polarimetry in the 1 arcmin field of view, four-color photometry and polarimetry of object and comparison star simultaneously in the 15 arcsec field, spectroscopy and the spectropolarimetry with 1-5 arcsec slit. It allows to measure the 3 Stokes parameters simultaneously, and is able to study $20-22^{\mathrm{m}}$ objects for 1-hour exposure under good weather conditions.

In summer 2006 we performed the set of observations of two objects-candidates to isolated stellar-mass black holes, the MACHO-99-BLG-22 and the J1942+10.

The MACHO-99-BLG-22 is the longest microlensing event with $1120 \pm 90$ days duration, projected velocity of $75 \pm 8 \mathrm{~km} / \mathrm{s}$, and the baseline magnitude $I=19.2^{\mathrm{m}}$ (Bennett et al. 2002). The analysis of microlensing data provided three possible models of the lens, with masses of $130 M_{\odot}\left(\right.$ at $0.5 \mathrm{kpc}$ with $\left.V_{t}=70 \mathrm{~km} / \mathrm{s}\right), 30 M_{\odot}$ (at $2 \mathrm{kpc}$ with 

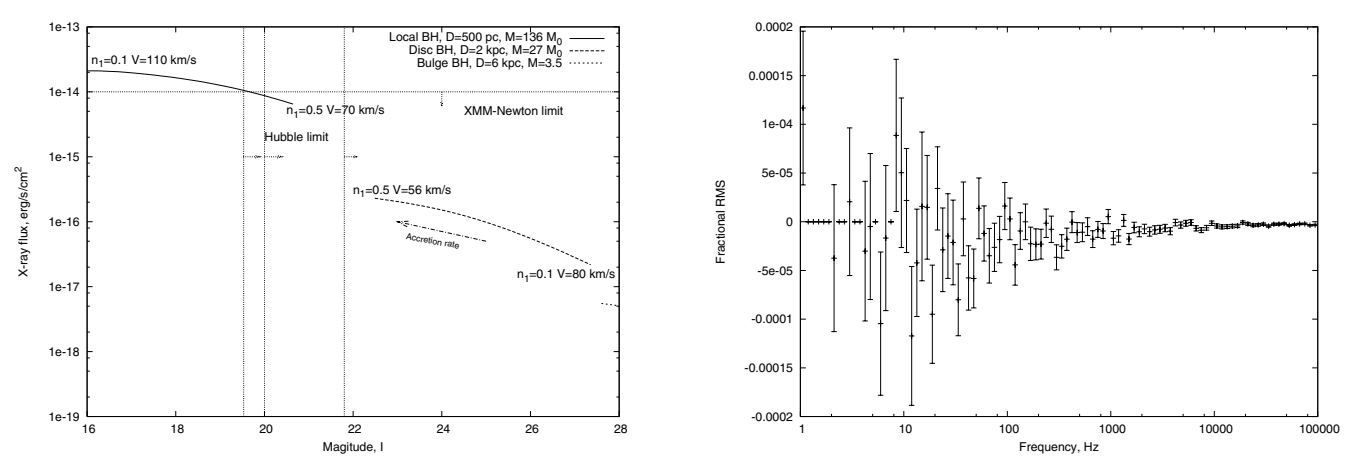

Figure 4. Left panel: the limits for different models of the BH parameters for the MACHO-99-BLG-22 object from the x-ray and optical observations. Right panel: the optical Fourier power spectrum for the radio-loud x-ray source with featureless optical spectrum J1942+10. The upper limit for the variable emission component is $10 \%$ in $10^{-5}-1 \mathrm{~s}$ range.

$\left.V_{t}=56 \mathrm{~km} / \mathrm{s}\right)$ and $3.5 M_{\odot}\left(\right.$ at $\left.6 \mathrm{kpc}, V_{t}=19 \mathrm{~km} / \mathrm{s}\right)$. We placed the upper limit on the variability of the emission from the position of the object on the level of $B<21.5^{\mathrm{m}}(3 \sigma)$ over the $10^{-5}-1 \mathrm{~s}$ time scales. The combination of the I-band Hubble data $\left(I<20^{\mathrm{m}}\right)$ and XMM-Newton (flux ; $10^{-14} \mathrm{erg} / \mathrm{s} / \mathrm{cm}^{2}$ ) with our theoretical estimations for possible models (Beskin \& Karpov 2005) allow us to rule out the first model (see Fig. 4).

The J1942+10 is the radio-loud $\mathrm{x}$-ray object with featureless optical spectrum with $B \sim 18^{\mathrm{m}}$. We observed it for 40 minutes in photometric mode and placed the upper limits on the B-band variability on the $10 \%$ level over the $10^{-5}-1 \mathrm{~s}$ time scale (see Fig. 4 ).

\section{Conclusions}

In the near future we are planning to continue the investigation of the long MACHO events, radio-loud x-ray sources and ROCOSes.

For the increase of the efficiency of search for fast variability we are now developing the new generation of position-sensitive detector, with GaAs photocathode, and 16-electrode collector. It will have the quantum efficiency up to $30 \%$ in the $4000-8500$ A range, and spatial resolution of $20 \mu \mathrm{m}$ for the $10^{6}$ number of pixels.

\section{Acknowledgements}

This work has been supported by the INTAS (grant No 04-78-7366), Russian Foundation for Basic Research (grant No 04-02-17555) and by the Russian Science Support Foundation.

\section{References}

Bennett, D. P. et al. 2002, ApJ, 579, 639

Beskin, G. M., Komarova, V. N., Neizvestny, S. I. et al. 1997, ExA, 7, 413

Beskin, G. M. \& Tuntsov, A. V. 2002, A\&A, 394, 489

Beskin, G. M. \& Karpov, S. V. 2005, A\&A, 440, 223

Chakrabarti, S. K. 1996, PhysRep, 266, 229

Cherepashchuk, A. M. 2003, Usp.Fiz.Nauk, 173, 345

Debur, V. et al. 2003, Nuclear Instruments and Methods in Physics Research, A 513, 127.

Greiner, J., Cuby, J.-G. \& Mc Caughrean, M. J. 2001, Nature, 414, 522

Ipser, J. R. \& Price, R. H. 1982, ApJ, 255, 654

Oppenheimer, J.\& Snyder, H. 1939, Phys. Rev., 56, 455 
Pustilnik, S. A. 1977, Soobsch. SAO, 18, 3

Plokhotnichenko, V. et al. 2003, Nuclear Instruments and Methods in Phys. Res., A 513, 167.

Shields, G. A. 1999, PASP, 111, 661

Shvartsman, V. F. 1971, AZh, 48, 479

Shvartsman, V. F. 1977 Soobsch. SAO, 19, 3

Shvartsman, V. F., Beskin, G. M. \& Pustilnik, S. A. 1989, AFz, 31, 457

Shvartsman, V. F., Beskin, G. M. \& Mitronova, S. N. 1989, Astron. Report Letters, 15, 145

Tsarevsky, G. et al. 2005, A\&A, 438, 949

Will, C. M. 1998, gr-qc/9811036

MARGARITA SAFOnOva: When did you observe the reported MACHO event, 99-BLG-22, and how did you look for the lens?

Gregory Beskin: We have searched the field around the reported MACHO event two years after its occurrence and found three candidates for source stars. We also found an unidentified object that is quite possibly the moving lens for that event. 


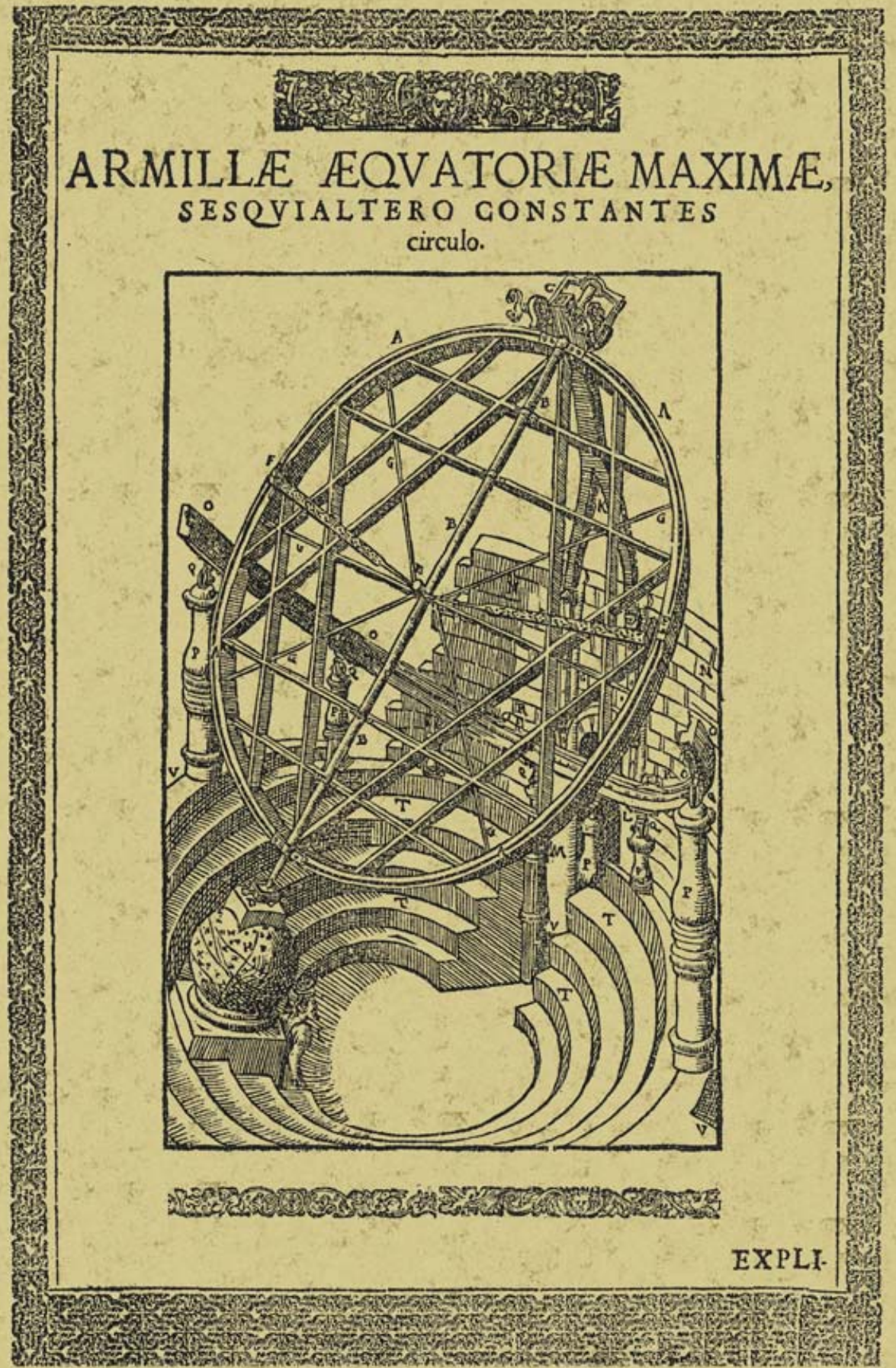

